\title{
ANÁLISE COMPARATIVA DE DADOS E PRODUTOS DE SENSORIAMENTO REMOTO COM RESOLUÇÃO ESPACIAL MODERADA PARA DETECÇÃO DE ÁREAS QUEIMADAS NO PARQUE NACIONAL DE ILHA GRANDE ENTRE 2007 E 2009
}

\author{
COMPARATIVE ANALYSIS OF REMOTE SENSING DATA AND PRODUCTS \\ WITH MODERATE SPATIAL RESOLUTION FOR DETECTION OF BURNED \\ AREAS IN THE ILHA GRANDE NATIONAL PARK BETWEEN 2007 AND 2009
}

\author{
ANÁLISIS COMPARATIVO DE DATOS Y PRODUCTOS DE \\ SENSORAMIENTO REMOTO CON RESOLUCIÓN ESPACIAL \\ MODERADA PARA DETECCIÓN DE ÁREAS QUEMADAS EN EL \\ PARQUE NACIONAL DE ISLA GRANDE ENTRE 2007 Y 2009
}
Everton Hafemann Fragal - Universidade Estadual de Maringá - Maringá - Paraná - Brasil evertonhaf@gmail.com
Nelson Vicente Lovatto Gasparetto - Universidade Estadual de Maringá - Maringá - Paraná - Brasil gasparetto31@gmail.com

\begin{abstract}
Resumo
Para entender a dinâmica de queimadas do Parque Nacional de Ilha Grande (PNIG), dados históricos de sensoriamento remoto são uma excelente alternativa. A análise do desempenho desses dados para detectar queimadas é importante a fim de minimizar erros de omissão e comissão. 0 objetivo desse estudo foi analisar o desempenho do dado MOD09 e produtos Fire_cci e MCD45, para detecção de queimadas no Parque Nacional de llha Grande, em comparação com o dado Landsat entre 2007 e 2009. Com base no dado MOD09 e produtos Fire_cci e MCD45 foram mapeadas as áreas queimadas e comparadas com o mapeamento de queimadas pelo dado Landsat. Como resultado, o dado MOD09 mostrou boa concordância espacial no mapeamento de queimadas, comparado com o dado Landsat (Kappa $=0,88$, erro de quantidade $=2,9 \%$ e erro de alocação $=$ $2,8 \%$ ), assim como, valores e distribuição de áreas queimadas (sobrestimativa média de 10\%). 0 dado MOD09 apresentou melhor desempenho na detecção de queimadas e é adequado para reconstruir historicamente a dinâmica de queimadas do parque. Os produtos Fire_cci e MCD45 não apresentaram bons desempenhos na detecção de queimadas, não sendo satisfatórios para entender a dinâmica de queimadas do parque.

Palavras-chave: Unidade de Conservação (UC), fogo, Gestão de UC e Sensoriamento Remoto.
\end{abstract}

\section{Abstract}

To understand dynamic of burned areas at llha Grande National Park (BINP) remote sensing historical data are excellent alternative. The analyze performance this data for detection burned areas is important to minimize omission and commission errors. Herein, this study analyzed the performance of MOD09 data and Fire_cci and MCD45 products to detect burned areas at Big Island National Park compared to Landsat data between 2007 and 2009. MOD09 data, Fire_cci and MCD45 products were used to map burned areas and compared with burned areas mapped by Landsat data. These investigation demonstrated, MOD09 data showed good spatial agreement in burned area mapping compared with Landsat data (Kappa $=0.88$, quantity disagreement $=2.9 \%$ and allocation disagreement $=2.8 \%$ ) as well as burned area values and distribution of burned area 
(mean overestimated of 10\%). MOD09 data showed better performance in detect burned area and is suitable to historical reconstruction the dynamic of burned areas at park. The Fire cci and MCD45 products do not presenter good performances to detect burned areas and are not suitable to understand the dynamic of burned areas at park.

Keywords: Conservation Unit (CU), fire, CU Management and Remote Sensing.

\section{Resumen}

Para entender la dinámica de las quemas del Parque Nacional de Isla Grande (PNIG), los datos históricos de sensoramiento remoto son una excelente alternativa. El análisis del rendimiento de esos datos para detectar quemas es importante para disminuir los errores de omisión y comisión. El objetivo de este estudio fue analizar el comportamiento de datos MOD09 y productos Fire_cci y MCD45 para detectar incendios en el Parque Nacional de Isla Grande, en comparación con datos Landsat entre los años 2007 y 2009. Basados en los datos MOD09 y productos Fire cci y MCD45 fueron mapeadas las áreas quemadas y comparadas con el mapeado elaborado con los datos Landsat. Como resultado, el dato MOD09 mostró buena correlación espacial en el mapeo de quemas, comparándolo con el Landsat (Kappa $=0,88$, cantidad de error $=2,9 \%$ y asignación de error $=2,8 \%$ ), así como, valores y distribución de áreas quemadas (media estimada del 10\%). El dato MOD09 presentó mejor desempeño en la detección de zonas de quemas y es apropiado para reconstruir históricamente la dinámica de quemas del parque. Los productos Fire cci y MCD45 no mostraron buenos resultados en la detección de incendios, no siendo adecuados para entender la dinámica de quemas del parque.

Palabras claves: Unidad de Conservación (UC), fuego, Gestión de UC y Sensoramiento Remoto.

Introdução

A Unidade de Conservação (UC) de Proteção Integral, Parque Nacional de Ilha Grande (PNIG), tem ocorrências de queimadas antes mesmo da sua criação em 1997. O parque é um trecho da planície de inundação do alto curso do rio Paraná, que ainda tem capacidade de sustentar diversidade ecológica elevada (Koproski et al., 2006). As queimadas ameaçam a integridade do parque, que promovem impactos ambientais como redução da diversidade de animais e plantas, diminuição da fertilidade do solo e causam poluição atmosférica afetando diretamente a população local (Ramsar, 2005; MMA, 2007; Piovesan et al., 2013).

Entender a dinâmica de queimadas no parque auxilia a organização dos recursos humanos e financeiros para o combate de novas queimadas e na investigação dos seus efeitos ao meio ambiente e sociedade (Costa; Dean, 2002). Para tal, é necessário o mapeamento histórico das cicatrizes de queimadas, que são caracterizadas pelas áreas com depósitos de carvão e cinzas provenientes da combustão da cobertura vegetal. Dados históricos de sensoriamento remoto são importantes para o mapeamento de queimadas, como da série temporal de imagens Landsat 5, 7 e 8, sensores TM, ETM+ e OLI, respectivamente. A partir da família de satélites Landsat, há disponibilidade de imagens compatíveis de comparação desde 1984 ao presente (2017). 
O dado Landsat é adequado para mapear áreas queimadas devido à resolução espacial de $30 \mathrm{~m}$. Entretanto, o dado Landsat tem resolução temporal de 16 dias, ou seja, sem alta frequência temporal (e.g. diária) para o mapeamento de queimadas. Desta forma, queimadas recentes (e.g. 1 a 5 dias após a queimada) não são detectadas, caso não coincida com o dia de imagemento do satélite Landsat.

A partir da década de 2000 muitos dados de sensoriamento remoto com resolução espacial moderada (e.g. 100 a 500 m) e resolução temporal alta (e.g. diário próximo ao Equador) foram disponibilizados para a comunidade em geral. Essas novas fontes de dados permitem com certa precisão espacial, o aumento da frequência temporal do mapeamento de queimadas, em relação ao realizado com o dado Landsat.

Nessa perspectiva alguns dados de sensoriamento remoto mostram ter potencial para o mapeamento de queimadas do parque, como o MODIS MOD09, que possui resolução espacial de $250 \mathrm{~m}$ e produtos globais de áreas queimadas, como o Fire_cci, e MODIS MCD45 com resoluções espaciais de 300 e 500 m, respectivamente. A descrição desses dados de sensoriamento remoto se encontra na seção de materiais e métodos.

Esses dados de sensoriamento remoto de resolução espacial moderada possuem avaliações globais para detecção de queimadas, com tolerância maior aos erros de omissão e comissão por abrangerem dimensões continentais (Padilla; Stehman; Chuvieco, 2014; Roy et al., 2008). No entanto, pouco se conhece do desempenho desses dados de sensoriamento remoto com resolução espacial moderada para detecção de queimadas em regiões com pequena dimensão, como a do PNIG.

Em geral, os dados de sensoriamento remoto apresentam melhor desempenho na detecção de queimada, quanto maior for a resolução espacial. No entanto, essa relação tem grande influência da dimensão das queimadas, além de outros fatores (e.g. cobertura de nuvem). Assim, é prudente verificar o desempenho de dados de sensoriamento remoto com resolução espacial moderada para detecção de cicatrizes de queimadas, comparando com dados de resolução espacial maior, como o dado Landsat (Boschetti; Flasse; Brivio, 2004).

Nesse contexto, a hipótese desse estudo é de que o dado MOD09, produtos Fire_cci e MCD45 têm diferentes desempenhos para detectar cicatriz de queimadas do Parque Nacional de Ilha Grande, tendo o dado ou produtos com maior resolução espacial, maior desempenho na detecção de queimadas. 
Assim, esse estudo tem o objetivo de analisar o desempenho da detecção de cicatrizes de queimadas pelo dado MOD09 e produtos Fire_cci e MCD45 no Parque Nacional de Ilha Grande, comparando com o dado Landsat para os anos de 2007 a 2009.

Material e métodos

Área de estudo

A área de estudo é o PNIG que ocupa área de 76.000 ha e situa-se na divisa dos estados do Paraná e Mato Grosso do Sul (Figura 1). O PNIG está inserido na Área de Proteção Ambiental de Ilhas e Várzeas do rio Paraná (UC de Uso Sustentável).

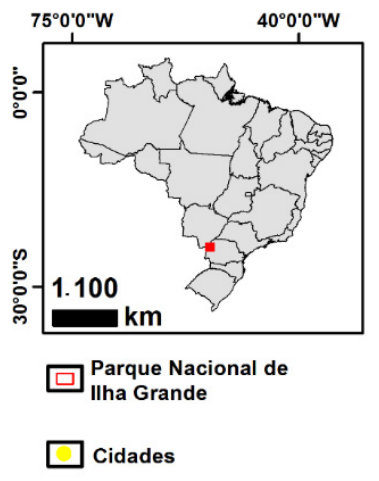

Datum horizontal: WGS 84 Fonte: IBGE (2017)

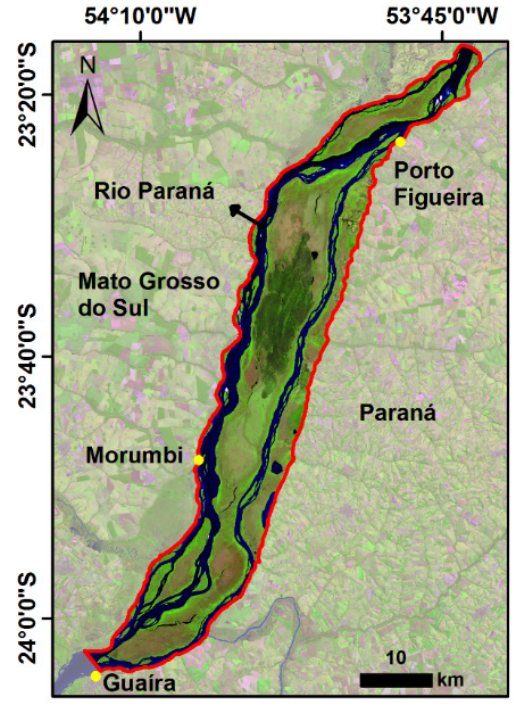

Figura 1 - Localização da área de estudo. Imagem Landsat de 22/06/2015 com composição colorida falsa R5 G4 B3

Fonte: Elaborado pelos autores (2016).

O clima da região do parque é caracterizado como Cfa, segundo a classificação de Köppen, que tem predominância de maior temperatura e concentração de precipitação na estação de verão, além de frequência de geada baixa e sem estação de seca definida (IAPAR, 2000). A precipitação acumulada anual varia de 1.400 a $1.600 \mathrm{~mm}$, com maiores concentrações nos meses de dezembro, janeiro e fevereiro (400 a $500 \mathrm{~mm}$ ), e menores nos meses de junho, julho e agosto (200 a $250 \mathrm{~mm}$ ). A temperatura média 
anual é de $24^{\circ} \mathrm{C}$, ocorrendo entre os meses de dezembro e fevereiro as maiores temperaturas médias de 29 a $30^{\circ} \mathrm{C}$ e entre os meses de junho e agosto, as menores, de 18 a $19^{\circ} \mathrm{C}$.

Entre os meses de junho e agosto, que correspondem ao período de menor precipitação e temperatura no parque, a média de focos de calor entre 1998 e 2016 foi de 16 focos, enquanto no período de dezembro a fevereiro (maior precipitação e temperatura) média de 6 focos. Dessa forma, no período de menor precipitação há uma maior tendência de ocorrências das queimadas. Para mais informações, no Apêndice A encontra-se um quadro com os dados de focos de calor mensal entre os anos de 1998 e 2016.

No parque, dois tipos de vegetação estão presentes: a Floresta Estacional Semidecidual Aluvial (FESA) e as Formações Pioneiras com Influência Flúvio Lacustre (FPIFL) (IBGE, 2012). A distribuição da vegetação é fortemente influenciada pelo revelo (e.g. feições geomorfológicas, como baixios e diques) e inundação/umidade (e.g. áreas encharcadas por água) (Souza Filho, 1993; ICMBio, 2008).

A FESA corresponde à vegetação arbórea, com altura total das árvores em cerca de 10 a 15 m, e os troncos com média do diâmetro a cima do peito (DAP), em cerca de 15 a $30 \mathrm{~cm}$. Nas áreas mais elevadas do parque (e.g. diques marginais) encontram-se predominantemente as árvores das espécies Sloanea guianensis, Gallesia integrifolia e Ficus obtusiuscula (ICMBio, 2008). Nas áreas de baixios (e.g. bacias de inundação), que são encharcadas por águas e que há presença de árvores, poucas diferentes espécies são encontradas, sendo predominante a Calophyllum brasiliense, Inga affinis e Cecropia pachystachya. Em áreas com relevos intermediários (entre os diques e baixios, como em paleodiques) e que há presença de árvores, situam-se predominantemente as espécies Protium heptaphyllum, Unonopsis lindmanii Guazuma ulmifolia.

A FPIFL consiste nos tipos de vegetação herbácea e arbustiva, predominantes na área do parque. A FPIFL encontra-se principalmente em relevos intermediários a baixos (e.g. paleocanal e bacia de inundação), com elevada umidade nos sedimentos ou encharcados por água. Esta vegetação é circundada pela FESA, isto é, nas bordas do parque situa-se a FESA e no interior a FPIFL. Na FPIFL, a vegetação herbácea tem altura em cerca de 1 a $2 \mathrm{~m}$, elevada quantidade de indivíduos e são principalmente das famílias Cyperaceae e Poaceae (ICMBio, 2008). Já a vegetação arbustiva, situa-se entremeada à vegetação herbácea, sem concentração de indivíduos, em geral tem altura inferior a 5 m, e a família predominante é a Onagraceae. 
As queimadas da vegetação do parque ocorrem por causas antrópicas, predominantemente para renovação de pequenas pastagens ou cultivos e por causas naturais como descargas elétricas (e.g. raio). Dos registros de ocorrências de queimadas entre 2000 e 2015, 53\% são por causas antrópicas, $15 \%$ por causas naturais e $32 \%$ por causas não conhecidas (ICMBio, 2015).

\section{Dado MODO9}

O dado MOD09 foi desenvolvido pelo projeto Sistema de Observação da Terra (Earth Observing System, EOS) da Administração Nacional da Aeronáutica e Espaço (National Aeronautics and Space Administration, NASA). Desse dado foi utilizada a imagem em reflectância de superfície na região espectral infravermelho próximo (841-876 nm), fornecida pela plataforma Terra, sensor MODIS. Este dado tem resolução espacial de 250 m e é disponibilizado diariamente ou por uma composição temporal de oito dias (e.g. 01 a oito dias do mês), pelo portal<http://reverb.echo.nasa. gov/reverb/> (Justice et al., 2002). O dado MOD09 para a área de estudo situa-se no tile h13v11.

Na detecção de cicatriz de queimadas foram selecionadas as imagens com composição temporal de oito dias que abrangem as datas de 18/07/2007, 27/12/2008 e 20/05/2009. Como não se conhece a data exata da ocorrência de queimada, o dado MOD09 com composição temporal de oito dias foi adequado para que não ocorra omissão na detecção de queimada.

\section{Produto Fire_cci}

O produto Fire_cci corresponde a um projeto de mapeamento global de áreas queimadas, desenvolvido pelo programa de Iniciativa a Mudanças Climáticas (Climate Change Initiative, CCI), coordenado pela Agência Espacial Européia (European Space Agency, ESA). Esse produto é baseado na combinação de dados entre uma série temporal de imagens em reflectância de superfície, na região espectral do infravermelho próximo do sensor MERIS, do satélite Envisat e imagens de focos de calor disponibilizado por produto MODIS.

Com resolução espacial de 300 m o produto Fire_cci tem resolução temporal diária, sendo disponibilizado por meio de uma composição 
temporal mensal (imagem que agrupa as ocorrências de queimadas de um mês), no portal <http://www.esa-fire-cci.org/> (Alonso-Canas; Chuvieco, 2015). Até o ano de 2016 esse produto é apenas disponibilizado para os anos de 2005 a 2011, porém existe a proposta de extensão dessa série temporal para os anos de 1980 a 2016. Esse produto abrange a área de estudo na região dois, segundo a classificação de regiões continentais do projeto Fire_cci (Pettinari et al., 2016).

O produto Fire_cci disponibiliza três subprodutos, sendo o primeiro, uma imagem que registra o dia juliano de ocorrência da queimada, o segundo, uma imagem com nível de confiabilidade de ocorrência da queimada e o terceiro, uma imagem com o uso e cobertura da terra queimada com base no mapa de uso e cobertura da terra Globcover 2005. O nível de confiabilidade varia de zero (baixa confiabilidade) a 100 (alta confiabilidade) para a ocorrência de queimada, em que foi selecionado o pixel com nível de confiabilidade a cima do valor 50.

Na detecção de queimadas foi selecionado o produto Fire_Cci, que compreende as datas de 18/07/2007, 27/12/2008 e 20/05/2009. Foram mapeados os pixels com ocorrência de queimada nas datas mencionadas com um desvio padrão de quatro dias devido à incerteza do dia exato de início da queimada.

\section{Produto MCD45}

O produto MCD45 foi desenvolvido por um projeto da NASA a fim de mapear globalmente áreas queimadas. O produto MCD45 consiste na estimativa de áreas queimadas por meio de diferentes bandas espectrais em reflectância de superfície do sensor MODIS (Roy et al., 2005). Esse produto apresenta resolução espacial de $500 \mathrm{~m}$, possui dado diário de queimada, sendo disponibilizado por meio de uma composição temporal mensal (agrupa as áreas queimadas de um mês), pelo portal <http:// reverb.echo.nasa.gov/reverb/> (Roy et al., 2008). Para a área de estudo esse produto situa-se no tile h13v11.

O produto MCD45 possui oito subprodutos, dentre os quais foram utilizados apenas o burn data (imagem com o dia juliano de ocorrência da queimada) e burn quality assessment (imagem com nível de confiabilidade da ocorrência de queimada). O nível de confiabilidade desse produto varia de um (maior confiabilidade) a cinco (menor confiabilidade). Foram 
selecionados apenas pixels com níveis de confiabilidade entre um e quatro, sendo descartados os pixels com o nível cinco devido apresentarem grandes incertezas na ocorrência de queimadas.

Foi utilizado o produto MCD45 para as datas de 18/07/2007, 27/12/2008 e 20/05/2009. Foram mapeados os pixels com ocorrência de queimadas nas datas mencionadas e com um desvio de quatro dias devido à incerteza do dia exato de queimada.

\section{Dado Landsat}

O dado Landsat corresponde a imagens multiespectrais para a observação dos objetos da superfície terrestre, que foi desenvolvido pela NASA. O dado Landsat utilizado nesse estudo corresponde a imagens em reflectância de superfície, na região espectral do infravermelho próximo (760-900 nm), do satélite Landsat, sensor TM. Esse dado possui resolução espacial de $30 \mathrm{~m}$ com disponibilização a cada 16 dias, sendo encontrado no portal < https://earthexplorer.usgs.gov/> (Wulder et al., 2012). Para abranger a área de estudo foi utilizado o dado Landsat das órbitas/ponto 224/076 e 224/077.

O dado Landsat foi utilizado para a análise do desempenho de detecção das queimadas pelo dado MOD09 e produtos Fire_cci e MCD45. Foram utilizados dados para as datas de 18/07/2007, 27/12/2008 e 20/05/2009, devido ter ocorridas queimadas no parque nesses anos. Para simplificar a referência às datas de 18/07/2007, 27/12/2008 e 20/05/2009, foram chamadas apenas pelos anos, sendo de 2007, 2008 e 2009, respectivamente.

\section{Mapeamento das cicatrizes de queimadas e validação}

Com o intuito de verificar a aparência visual, disposição e abrangência das cicatrizes de queimadas foram realizadas a interpretação visual dos dados Landsat e MOD09 e produtos Fire_cci e MCD45 para o ano de 2008. Na interpretação visual da cicatriz de queimada foram utilizados os elementos de cor, tamanho e forma (Jensen, 2009).

Foram mapeadas as cicatrizes de queimadas por meio da segmentação espacial e fatiamento linear dos valores espectrais do infravermelho próximo dos dados Landsat e MOD09. Essa região espectral foi adotada por ser adequada para discriminar áreas queimadas em relação as não queimadas (Ponzoni; Shimabukuro, 2007). 
Para os produtos Fire_cci e MCD45 o mapeamento das áreas queimadas ocorreram pela segmentação espacial e fatiamento linear dos dias julianos de ocorrências das queimadas, com desvio de quatro dias. Por exemplo, a data de 18/07/2007corresponde ao dia juliano 199, então, os pixels que apresentaram dias julianos entre 195 e 203 foram rotulados como áreas queimadas.

A validação dos mapeamentos de áreas queimadas foi realizada pelo índice Kappa, erro de quantidade (Quantity Disagreement, QD) e erro de alocação (Allocation Disagreement, $A D$ ) (Congalton, 1991; Pontius; Millones, 2011). O índice Kappa expressa o acerto da classe mapeada em análise (queimada mapeada pelo dado MOD09 e produtos Fire_cci e MCD45) em relação à classe mapeada de referência (queimada mapeada pela imagem Landsat), caso fosse de modo aleatório. O QD consiste pela diferença de quantidade da classe mapeada em análise em relação à classe de referência. O AD corresponde a diferença de posicionamento da classe mapeada analisada em relação a classe de referência.

Foi analisada a diferença espacial entre o mapeamento de áreas queimadas pelo dado MOD09 e produtos Fire_cci e MCD45 e o mapeamento de áreas queimadas pelo dado Landsat. Para tal, sobre a área de estudo foi criada uma grade regular de largura e comprimento de 1,5 km, com área de 225 ha. Para cada célula da grade com a presença de área queimada mapeada pela imagem Landsat, foi calculada a diferença com área queimada mapeada pelo dado MOD09 e produtos Fire_cci e MCD45, e espacializada.

\section{Resultados e discussão}

A interpretação visual da cicatriz de queimada do dado Landsat do ano de 2008, na região espectral do infravermelho próximo apresentou cor preta, com tamanho grande que abrangeu cerca de $70 \%$ da área do PNIG de modo contínuo e com forma irregular (Figura 2). Verifica-se no setor noroeste uma extensa e contínua cor branca que corresponde a presença de nuvens.

O dado MOD09 mostrou a cicatriz de queimada muito semelhante à imagem Landsat, sendo de cor preta, tamanho grande com modo contínuo e forma irregular. Nota-se no setor nordeste a presença de pixels de cor branca entre a área queimada que se caracteriza como ruído. Devido à combustão da cobertura vegetal e à presença de fuligem, a região espectral 
do infravermelho próximo (dados Landsat e MOD09) é pouca refletida ao sensor e, por sua vez, muito absorvida pelo material queimado que se destaca em relação aos outros objetos da cena (Ponzoni; Shimabukuro, 2007).

TM

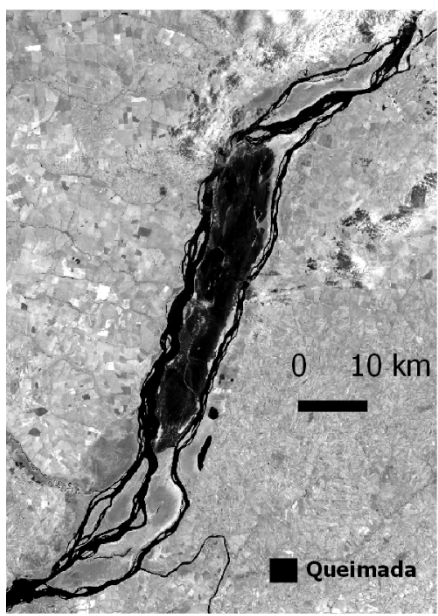

Fire_cci

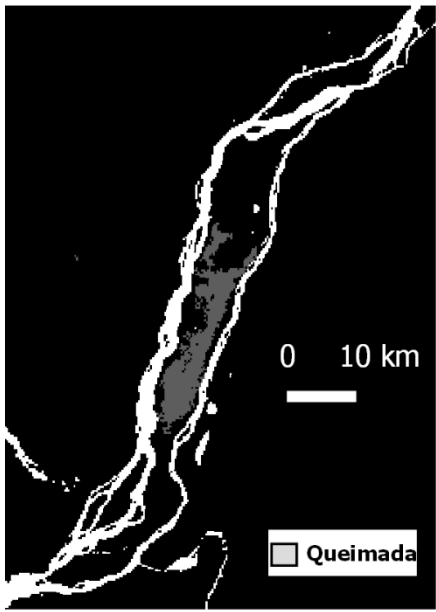

Figura 2 - Interpretação visual da cicatriz de queimada no PNIG no ano de 2008, por dados Landsat e MOD09 e produtos Fire_cci e MCD45
MOD09

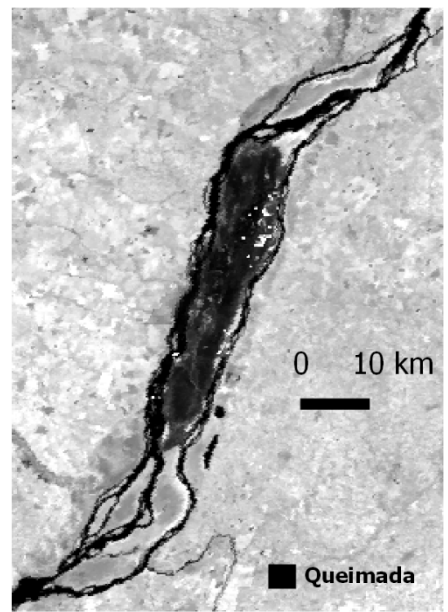

MCD45

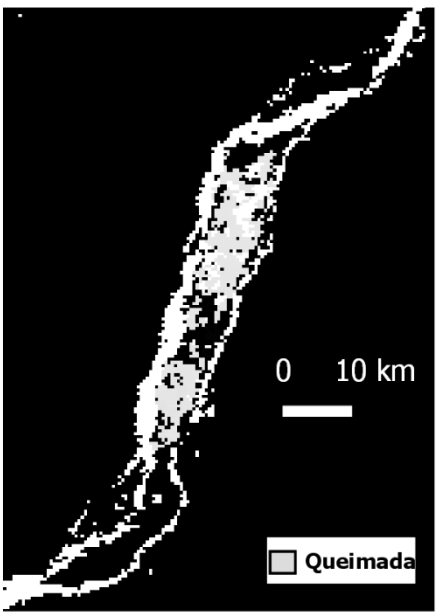

Fonte: Elaborado pelos autores (2016). 
O produto Fire_cci apresentou cicatriz de queimada com cor cinza-escuro, com tamanho menor em relação ao dado Landsat, ligeira fragmentação da área queimada e forma semirretangular. O produto MCD45 mostrou a cicatriz de queimada em cor cinza-claro com tamanho menor, quando comparada ao dado Landsat, fragmentação das áreas queimadas e forma irregular. Embora seja importante o elemento visual cor para interpretação dos dados Landsat e MOD09, para os produtos Fire_cci e MCD45 não é relevante, uma vez que, poderiam ser representados em qualquer paleta de cor.

Exceto pelo elemento de interpretação visual cor, as variações de tamanho e forma representam desconformidades na detecção de área queimada do dado MOD09 e produtos Fire_cci e MCD45, em relação ao dado Landsat. Essas desconformidades estão associadas predominantemente com as características de resolução espacial, da sensibilidade do sensor e algoritmo de processamento da detecção de queimadas (Chuvieco et al., 2016; Roy et al., 2008).

Com o uso de imagens Landsat como referência no mapeamento das cicatrizes de queimadas, o dado MOD09 apresentou maior semelhança visual, enquanto os produtos Fire_cci e MCD45 menor semelhança visual (Figura 3). No ano de 2007 a queimada no PNIG se concentrou no setor sul, sendo visualmente bem detectado pelo dado MOD09 e produto MCD45. Para o ano de 2008 a área queimada se concentrou no setor central do parque, apresentando grande extensão, apenas com boa representação visual pelo dado MOD09.

No ano de 2009 existiram duas áreas de concentração de queimadas, sendo a primeira no setor nordeste e a segunda no setor sudeste (Figura 3). Apenas o dado MOD09 apresentou capacidade de detecção nos dois setores de queimadas, no entanto, o produto MCD45 apresentou visualmente boa detecção da área queimada no setor sudeste. O produto Fire_cci mostrou visualmente pequena detecção das queimadas para os anos de 2007 a 2009. 

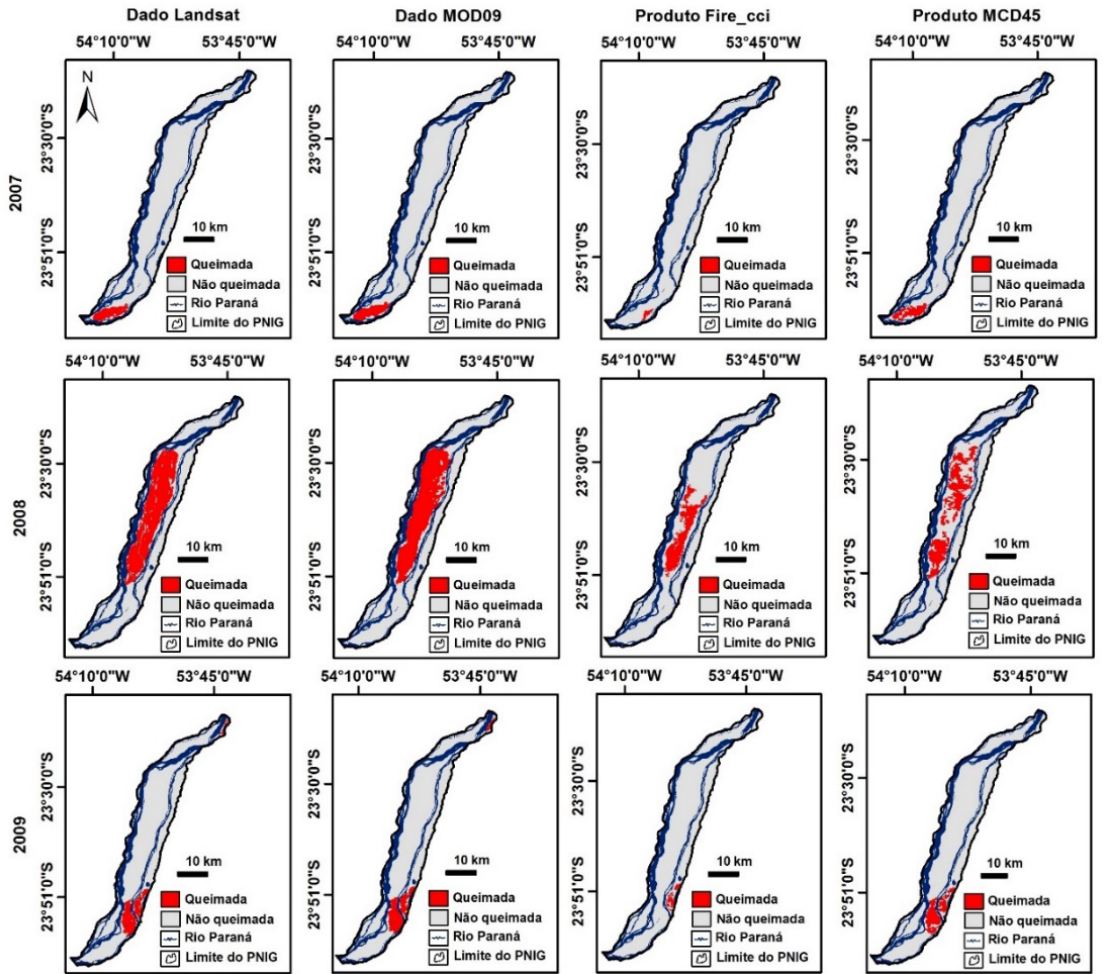

Figura 3 - Mapeamento de áreas queimadas no PNIG entre 2007 e 2009,com dados Landsat e MOD09 e produtos Fire_cci e MCD45

Fonte: Elaborado pelos autores (2016).

No ano de 2008 houve maior área queimada (28.822 ha) no parque, detectada pelo dado Landsat e menores áreas queimadas nos anos de 2009 (6.362 ha) e 2007 (3.515 ha), respectivamente (Figura 4). O dado MOD09 sobrestimou em média de $10 \%$ os valores de áreas queimadas, em relação àquelas mapeadas pelo dado Landsat nos anos de 2007 a 2009. Isso foi atribuído a sua resolução espacial $(250 \mathrm{~m})$ : cada pixel possui uma área cerca de oitos vezes maior que de um pixel Landsat (30 m). Dessa forma, mesmo que parte da área contida no pixel foi queimada no dado MOD09, foi registrado como se toda a área houvesse sido queimada. 


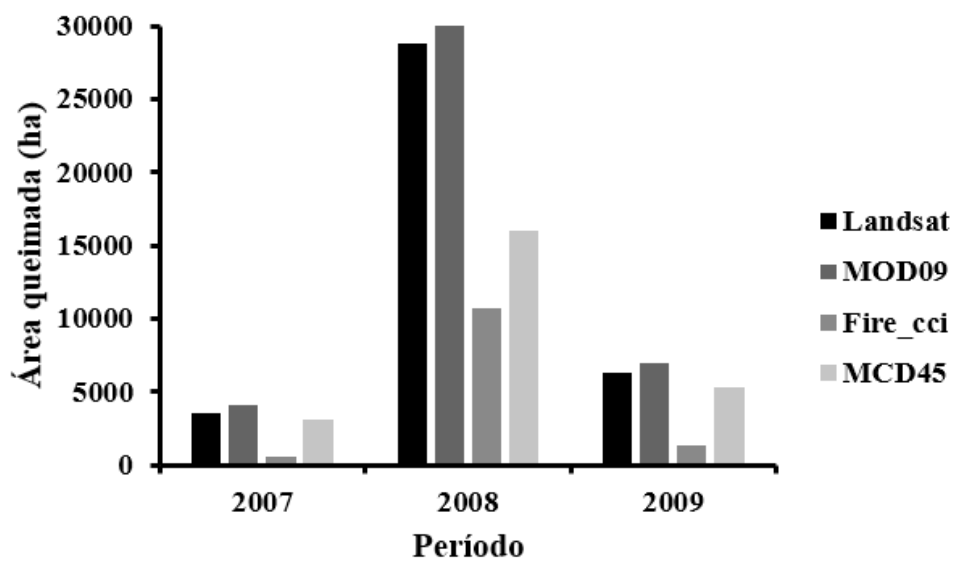

Figura 4 - Áreas queimadas dos anos de 2007 a 2009, estimadas por dados Landsate MOD09 e produtos Fire_cci e MCD45

Fonte: Elaborado pelos autores (2016).

O produto Fire_cci apresentou as menores áreas queimadas em relação ao dado Landsat, subestimando em média $75 \%$ os valores de áreas queimadas, para os anos de 2007 a 2009. O produto MCD45 apresentou ligeiramente áreas queimadas menores, comparado ao dado Landsat, que subestimou em média de $24 \%$ os valores de áreas queimadas para os anos analisados. Em relação aos valores de áreas queimadas, ambos os produtos não promoveram sobrestimativa.

A partir da análise conjunta dos valores e distribuição de áreas queimadas (Figuras 3 e 4), é evidente que o dado MOD09 e produtos Fire_cci e MCD45 apresentam diferentes capacidades de detecção das queimadas, sendo vinculadas com a extensão das cicatrizes de queimadas. Trabalhos que buscaram analisar o desempenho de produtos de sensoriamento remoto na detecção de queimadas, também verificaram que a extensão da queimada é um fator limitante, onde áreas pequenas são pouco detectadas (Piromal et al., 2008; Libonati et al., 2015).

A validação da concordância espacial do mapeamento das áreas queimadas pelos índices Kappa, QD e AD, com base no dado Landsat, mostrou que o dado MOD09 alcançou melhor resultado em relação aos produtos Fire_cci e MCD45, para os anos de 2007 a 2009 (Quadro1). Desta forma, em média o dado MOD09 apresentou índice Kappa de 0,87, QD de $2,9 \%$ e $\mathrm{AD}$ de $2,8 \%$. 
O mapeamento de áreas queimadas pelo produto Fire_cci apresentou pequeno valor do índice Kappa (média de 0,40) e principalmente grande valor do erro de quantidade (QD, média de 28\%). No mapeamento de áreas queimadas com base no produto MCD45, em geral, mostrou bom acerto espacial com um índice Kappa médio de 0,65 e pequeno valor de erro de alocação (em média menos de 1\%). No entanto, apresentou erro de quantidade médio de 15\%, considerado elevado devido à dimensão da área de estudo.

Quadro 1 - Índices de validação dos mapeamentos de áreas queimadas pelo dado MOD09 e produtos Fire_cci e MCD45, com base em dado Landsat entre os anos de 2007 e 2009. OD corresponde ao erro de quantidade (Quantity Disagreement) e AD pelo erro de alocação (Allocation Disagreement)

\begin{tabular}{|c|c|c|c|c|}
\hline & & & alidaç & \\
\hline Produtos & Período & Карра & OD & AD \\
\hline MOD09 & \multirow{3}{*}{2007} & 0,82 & 6,6 & 2,6 \\
\hline Fire_cci & & 0,55 & 40,8 & 0 \\
\hline MCD45 & & 0,72 & 10,8 & 0 \\
\hline MOD09 & \multirow{3}{*}{2008} & 0,92 & 1,4 & 2,7 \\
\hline Fire_cci & & 0,44 & 27,4 & 0 \\
\hline MCD45 & & 0,53 & 26,7 & 0 \\
\hline MOD09 & \multirow{3}{*}{2009} & 0,88 & 0,8 & 3,2 \\
\hline Fire_cci & & 0,22 & 16,4 & 0,8 \\
\hline MCD45 & & 0,69 & 7,6 & 0,8 \\
\hline
\end{tabular}

Fonte: Elaborado pelos autores (2016).

As diferenças espaciais de áreas queimadas mapeadas pelos dados MOD09 e Landsat, analisadas sob a grade regular, foram predominantemente mínimas (cerca de 10 ha), para os anos de 2007 a 2009 (Figura 5). As diferenças espaciais de áreas queimadas entre o produto Fire_cci e o dado Landsat, foram grandes, sendo que em muitas células da grade foram subestimadas até 225 ha. O produto Fire_cci subestimou áreas queimadas, principalmente nas regiões de transição entre áreas queimadas e não queimadas. As diferenças espaciais de áreas queimadas mapeadas pelo produto MCD45, em relação ao dado Landsat, foram grandes tanto para subestimar, quanto para sobrestimar. 
MOD09 X Landsat
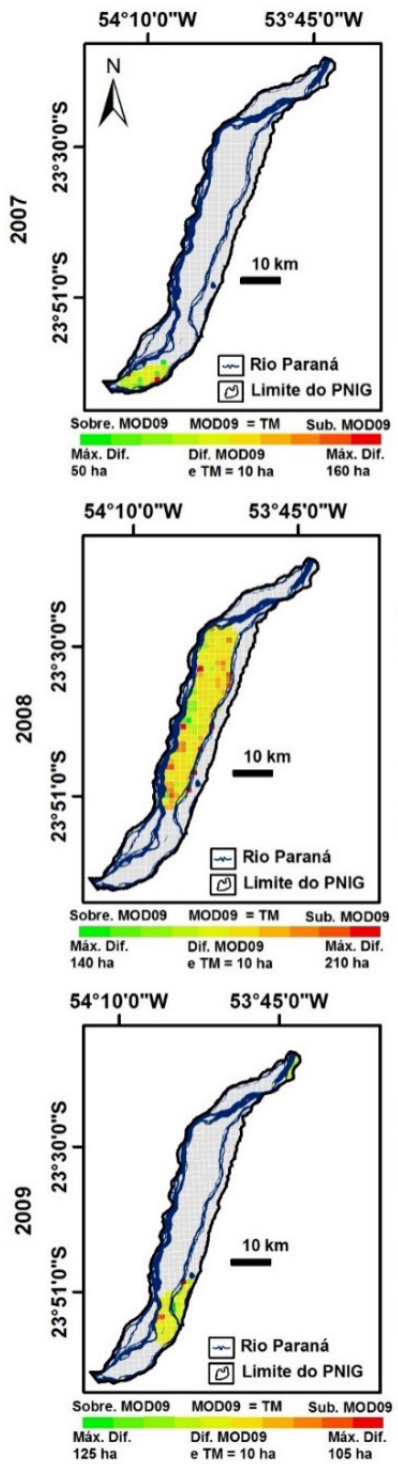

Fire_cci X Landsat

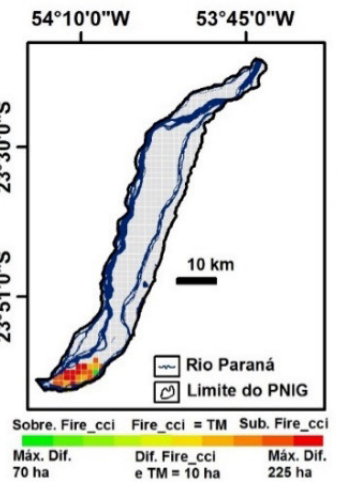

$54^{\circ} 10^{\prime} 0^{\prime \prime W} \quad 53^{\circ} 45^{\prime} \mathrm{O}^{\circ} \mathrm{W}$
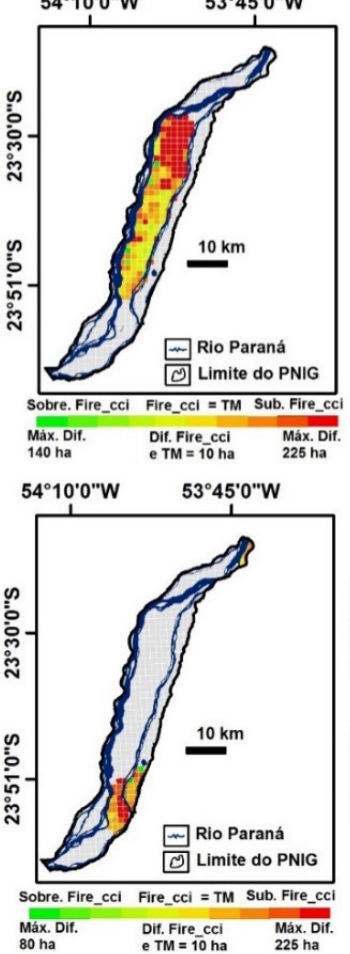

MCD45 X Landsat
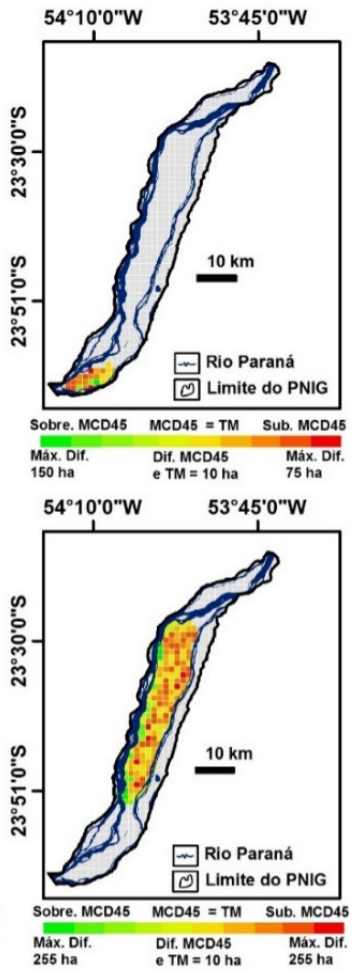

$54^{\circ} 10^{\prime} 0^{\prime \prime} \mathrm{W} \quad 53^{\circ} 45^{\prime} 0^{\prime \prime} \mathrm{W}$

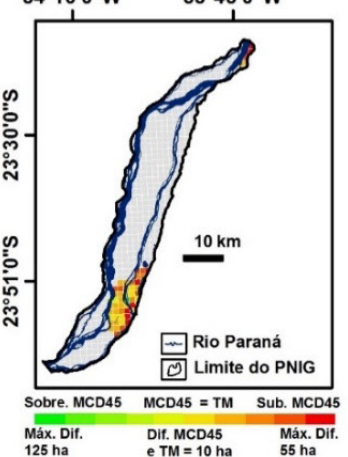

Figura 5 - Diferença de área queimada mapeada pelo dado MOD09 e produtos

Fire_cci e MCD45, em relação ao dado Landsat, para os anos de 2007 a 2009 
A partir da análise dos valores de área, concordância espacial do mapeamento e diferenças espaciais de áreas queimadas, constata-se que o dado MOD09 mostrou melhor desempenho para detecção de queimadas, em relação aos produtos Fire_cci e MCD45. Comparado ao dado Landsat para mapear queimadas, o dado MODo9 é adequado para reconstruir historicamente a dinâmica de queimadas no parque, a partir da década de 2000. Tal constatação foi tomada também por Lima et al. (2012) para um setor de floresta amazônica no estado do Pará, em que o dado MOD09 apresentou bom desempenho na detecção de queimadas e poucas diferenças espaciais no mapeamento de áreas queimadas, em relação ao dado Landsat.

Em relação ao produto Fire_cci foi analisado seu desempenho para o mapeamento de queimada para todo o globo terrestre, que mostrou bom desempenho, principalmente em relação ao produto global Global Fire Emission Database 4 (GFED4) (Chuvieco et al., 2016). No entanto, uma análise de desempenho de detecção de queimadas de uma área global, não é o suficiente para assegurar o bom desempenho da detecção de queimada em áreas específicas, como desse estudo.

O produto MCD45 não mostrou um desempenho satisfatório para detecção de queimadas no PNIG, no entanto, foi melhor que o produto Fire_cci. Embora possua valores de áreas queimadas parecidas com o dado Landsat, promoveu uma grande variação espacial de sub e sobrestimativa. Libonati et al. (2015) analisaram o produto MCD45 na região do Jalapão, estado do Tocantins, e obtiveram subestimativa de mais de $60 \%$ na detecção de queimadas, comparado com o dado Landsat. O produto MCD45 apresenta a característica de mapear globalmente áreas queimadas, o qual tem maior tolerância a erros de comissão e principalmente omissão.

\section{Considerações finais}

O dado MOD09 demonstrou maior desempenho na detecção das cicatrizes de queimadas no PNIG entre os anos de 2007 a 2009 em relação aos produtos Fire_cCi e MCD45. Portanto, o dado MOD09 é adequado para entender a dinâmica de queimadas no PNIG. Esse resultado confirma a hipótese que o dado de sensoriamento remoto com resolução espacial maior possui maior desempenho na detecção de cicatrizes de queimadas. No entanto, a hipótese não foi confirmada em relação aos produtos 
analisados, sendo o MCD45 com resolução espacial de $500 \mathrm{~m}$, obteve melhor resultado que o Fire_cci (300 m).

\section{Referências}

ALONSO-CANAS, I.; CHUVIECO, E. Global Burned Area Mapping from ENVISAT-MERIS data. Remote Sensing of Environment, v.163, p. 140-152, 2015.

BOSCHETTI, L.; FLASSE, S. P.; BRIVIO, P. A. Analysis of the conflict between omission and commission in low spatial resolution dichotomic thematic products: The Pareto Boundary. Remote Sensing of Environment, v. 91, p. 280292, 2004.

CHUVIECO, E. et al. A new global burned area product for climate assessment of fire impacts. Global Ecology and Biogeography, v. 25, p. 619-629, 2016.

CONGALTON, R. G. An review of assessing the accuracy of classifications of remotely sensed data. Remote Sensing of Environment, v. 49, n. 12, p. 1671-1678, 1991.

COSTA, E. P.; DEAN, W. A Ferro e Fogo: A história da devastação da Mata Atlântica brasileira. São Paulo: Companhia das Letras, 2002. 484 p.

INSTITUTO AGRONÔMICO DO PARANÁ (IAPAR). Cartas Climáticas do estado do Paraná. 2000. Disponível em: <http://www.pr.gov.br/iapar/sma/ Cartas_Climáticas/Cartas_Climáticas.htm>. Acesso em: 5 jan. 2017.

INSTITUTO BRASILEIRO DE GEOGRAFIA E ESTATÍSTICA (IBGE). Manual Técnico da Vegetação Brasileira. 2012. Disponível em: <http://biblioteca.ibge. gov.br/visualizacao/livros/liv63011.pdf >. Acesso em: 30 out. 2016.

INSTITUTO CHICO MENDES DE CONSERVAÇÃO DA BIODIVERSIDADE (ICMBio). Plano de Manejo: Parque Nacional de Ilha Grande. 2008. Disponível em: < http://www.icmbio.gov.br/portal/images/stories/docs-planos-de-manejo/ parna_ilha_grande_pm.pdf $>$. Acesso em: 05 jul. 2015.

INSTITUTO CHICO MENDES DE CONSERVAÇÃO DA BIODIVERSIDADE (ICMBio). Plano operativo de prevenção e combate aos incêndios florestais do Parque Nacional de Ilha Grande - PR/MS. 2015. Disponível em: <http://www. icmbio.gov.br>. Acesso em: 05 jul. 2015.

JENSEN, J. R. Sensoriamento Remoto do Ambiente: Uma Perspectiva em Recursos Terrestres. Tradução: Epiphanio, José Carlos Neves, Formaggio, Antonio R. Santos, Athos R. Rudorff, Bernardo F. T. Almeida, Cláudia M. Galvão, Lênio S. São José dos Campos: Parêntese, 2009.

JUSTICE, C. O. et al. The MODIS fire products. Remote Sensing of Environment, v. 83, n. 1-2, p. 244-262, 2002.

KOPROSKI, L. P. et al. Impactos do fogo sobre serpentes (Squamata) no Parque Nacional de Ilha Grande (PR/MS), Brasil. Arquivos de Ciências Veterinárias e Zoologia da UNIPAR, Umuarama, v. 9, p. 129-133, 2006. 
LIBONATI, R. et al. An algorithm for burned area detection in the Brazilian Cerrado using 4 mm MODIS imagery. Remote Sensing, v. 7, p. 15782-15803, 2015.

LIMA, A. et al. Land use and land cover changes determine the spatial relationship between fire and deforestation in the Brazilian Amazon. Applied Geography, v. 34, p. 239-246, 2012.

MINISTÉRIO DO MEIO AMBIENTE (MMA). Áreas Prioritárias para a Conservação, Uso Sustentável e Repartição de Benefícios da Biodiversidade Brasileira: Atualização Portaria MMA n. 09 de 23 de janeiro de 2007. 2007. Disponível em: <http://www.mma.gov.br/estruturas/chm/_arquivos/ biodiversidade31.pdf >. Acesso em: 22 maio 2017.

PADILLA, M.; STEHMAN, S. V.; CHUVIECO, E. Validation of the 2008 MODISMCD45 global burned area product using stratified random sampling. Remote Sensing of Environment, v. 144, p.187-196, 2014.

PETTINARI, M. L. et al. ESA CCI ECV Fire disturbance: product user guide, version 2.1. 2016. Disponível em: <http://www.esa-fire-cci.org/documents>. Acesso em: 30 out. 2016.

PIOVESAN, J. C. et al. Processos ecológicos e a escala da paisagem como diretrizes para projetos de restauração ecológica. Revista CAITITU, n. 1, p. 5772, 2013.

PIROMAL, R. A. S. et al. Utilização de dados MODIS para detecção de queimadas na Amazônia. Acta Amazonica, v. 38, p. 77-84, 2008.

PONTIUS, R.G. JR.; MILLONES, M. Death to Kappa: birth of quantity disagreement and allocation disagreement for accuracy assessment. International Journal of Remote Sensing, v. 32, n. 15, p. 4407-4429, 2011.

PONZONI, F. J.; SHIMABUKURO, Y. E. Sensoriamento Remoto no Estudo da Vegetação. São José dos Campos: Parêntese, 2007. 144p.

RAMSAR. Resolution IX.4: Wetlands and poverty reduction. 2005. Disponível em: $\quad$ http://archive.ramsar.org/cda/en/ramsar-documents-resol-resolutionix-14/main/ramsar/1-31- 107\%5E23485_4000_0__>. Acesso em: 22 maio 2017.

SOUZA FILHO, E. E. Aspectos da geologia e estratigrafia dos depósitos sedimentares do rio Paraná entre Porto Primavera (MS) e Guaíra (PR). 1993. 214 f. Tese (Doutorado) - Instituto de Geociências da USP, São Paulo, SP, 1993.

ROY, D. P. et al. Prototyping a global algorithm for systematic fire affected area mapping using MODIS time series data. Remote Sensing of Environment, v. 97, p. 137-162, 2005.

ROY, D. P. et al. The Collection 5 MODIS Burned Area Product - Global Evaluation by Comparison with the MODIS Active Fire Product. Remote Sensing of Environment, v. 112, p. 3690-3707, 2008.

WULDER, M. A. et al. Opening the archive: How free data has enabled the science and monitoring promise of Landsat. Remote Sensing of Environment, v. 122, p. 2-10, 2012. 


\section{Apêndice 1 - Focos de calor do PNIG}

Quadro 1 - Número de focos de calor mensal entre os anos de 1998 e 2016 no PNIG

\begin{tabular}{|c|c|c|c|c|c|c|c|c|c|c|c|c|}
\hline Ano & Jan. & Fev. & Mar. & Abril & Maio & Jun. & Jul. & Ago. & Set. & Out. & Nov. & Dez. \\
\hline 1998 & 0 & 0 & 0 & 0 & 0 & 0 & 0 & 7 & 2 & 0 & 0 & 0 \\
\hline 1999 & 0 & 0 & 0 & 0 & 2 & 0 & 8 & 40 & 44 & 2 & 12 & 0 \\
\hline 2000 & 7 & 18 & 4 & 2 & 1 & 4 & 0 & 2 & 2 & 1 & 0 & 1 \\
\hline 2001 & 0 & 0 & 0 & 0 & 2 & 0 & 3 & 4 & 5 & 0 & 0 & 0 \\
\hline 2002 & 1 & 2 & 0 & 13 & 0 & 0 & 0 & 14 & 46 & 2 & 0 & 0 \\
\hline 2003 & 1 & 0 & 0 & 0 & 0 & 2 & 7 & 11 & 96 & 0 & 2 & 0 \\
\hline 2004 & 2 & 1 & 1 & 12 & 0 & 0 & 0 & 3 & 2 & 0 & 0 & 0 \\
\hline 2005 & 1 & 1 & 193 & 8 & 4 & 5 & 1 & 66 & 1 & 1 & 1 & 1 \\
\hline 2006 & 1 & 10 & 4 & 10 & 508 & 1 & 1 & 106 & 1 & 2 & 0 & 0 \\
\hline 2007 & 5 & 4 & 6 & 0 & 0 & 13 & 67 & 4 & 28 & 94 & 10 & 6 \\
\hline 2008 & 0 & 0 & 2 & 15 & 9 & 4 & 11 & 52 & 0 & 0 & 5 & 133 \\
\hline 2009 & 0 & 0 & 4 & 79 & 37 & 6 & 0 & 2 & 0 & 1 & 3 & 2 \\
\hline 2010 & 1 & 4 & 6 & 4 & 3 & 1 & 8 & 63 & 29 & 8 & 8 & 2 \\
\hline 2011 & 4 & 1 & 1 & 0 & 6 & 7 & 4 & 17 & 7 & 0 & 0 & 6 \\
\hline 2012 & 3 & 3 & 14 & 1 & 2 & 0 & 18 & 13 & 238 & 281 & 9 & 35 \\
\hline 2013 & 4 & 1 & 2 & 0 & 5 & 0 & 4 & 255 & 6 & 5 & 12 & 1 \\
\hline 2014 & 11 & 6 & 95 & 0 & 0 & 1 & 4 & 6 & 2 & 29 & 2 & 2 \\
\hline 2015 & 49 & 18 & 16 & 5 & 0 & 7 & 2 & 16 & 12 & 7 & 47 & 0 \\
\hline 2016 & 4 & 9 & 5 & 3 & 1 & 3 & 6 & 43 & 12 & 1 & 29 & 5 \\
\hline
\end{tabular}

Fonte: INPE (2016).

Everton Hafemann Fragal - Doutorando em Geografia pela Universidade Estadual de Maringá (UEM). Mestre em Sensoriamento Remoto pelo Instituto Nacional de Pesquisas Espaciais (INPE). Bacharel e licenciado em Geografia pela UEM.

Nelson Vicente Lovatto Gasparetto - Graduado em Geologia pela Universidade do Vale do Rio dos Sinos, mestre em Geociências pela Universidade Federal do Rio Grande do Sul e doutor em Geociências pela Universidade de São Paulo. Atualmente é Bolsista Produtividade em Pesquisa do CNPq nível 2, professor do Departamento de Geografia e coordenador do Grupo de Estudos Multidisciplinares do Ambiente - GEMA/UEM da Universidade Estadual de Maringá - Paraná (PR). 
Contribuições dos autores

Os dois autores contribuíram substancialmente no desenvolvimento do artigo. O primeiro autor adquiriu, organizou e processou os dados e produtos utilizados no trabalho. Além disso, preparou e redigiu o manuscrito. O segundo autor contribuiu pela orientação do primeiro autor na construção do artigo e revisão crítica.

Recebido para publicação em 11 de abril de 2017. Aceito para publicação em 29 de maio de 2017. 